\title{
Seven Decades of Ascent in Superconductivity
}

\section{Ivan Božović ${ }^{1,2}$}

Published online: 26November 2020

(C) Springer Science+Business Media, LLC, part of Springer Nature 2019
On 20 January 2020, Theodore H. Geballe is turning 100. This extraordinary and precious occasion calls for a celebration in the condensed matter physics and materials science communities. Both of these have already presented Ted with their highest laurels. In 1970, the American Physical Society bestowed upon him the Oliver E. Buckley Solid State Physics Prize "for experiments that challenged theoretical understanding and opened up the technology of high-field superconductors." In 1989, he got the inaugural Bernd T. Matthias Memorial Award for innovative contributions to the material aspects of superconductivity. In 1991, the Materials Research Society presented Ted with the Von Hippel Award for "ingenious use of chemical principles to synthesize novel materials of technological importance, careful experiments on a wide range of materials to illuminate fundamental materials properties and behavior, and leadership in helping to formulate the modern concepts of interdisciplinarity as a scientist, teacher, and administrator." While Ted has made seminal contributions to various areas, including semiconductors and magnetism, most of his monumental opus has been devoted to superconductivity, the field in which he has played the lead role for seven decades. It is thus most fitting that this special issue of the Journal of Superconductivity and Novel Magnetism is devoted to Ted's 100th anniversary. No one was honored on such an occasion before, and it is unlikely that anyone else will be anytime soon.

Ted got his B.Sc. in Chemistry in 1941 at the University of California at Berkeley, under the supervision by William Giauque. Ted's studies were interrupted by the Second World War. He enlisted in the US Army and served, as an ordnance officer, in the Pacific theater-Australia, New

Ivan Božović

bozovic@bnl.gov

1 Brookhaven National Laboratory, Upton, New York 11973-5000, USA

2 Chemistry Department, Yale University, New Haven, CT 06520, USA
Guinea, and the Philippines. The USA and the allies won the war eventually, and Ted returned to Berkeley, as a graduate student. In 1949, he received his Ph.D. degree in Physical Chemistry, defending his thesis "Thermodynamic and Magnetic Properties of Single Crystal Cupric Sulfate Pentahydrate Below 4 Degrees Kelvin"-and his mentor, Giauque, received a Nobel Prize in Chemistry. Ted stayed at Berkeley as a postdoc for few more years and published his first research papers, including four with Giauque.

Then, in 1952, Ted moved to Bell Laboratories, the foremost US research center for solid state physics, as a staff member. Collaborators and coauthors on his early Bell-Lab papers include G. W. Hull, F. J. Morin, John Ziman, Conyers Herring, John Hulm, and Bernd Matthias. Already in 1954, together with Matthias, Ted discovered superconductivity in $\mathrm{Nb}_{3} \mathrm{Sn}$ with $T_{c}=18.3 \mathrm{~K}$. Remarkably, this compound remained superconducting in magnetic fields up to $30 \mathrm{~T}$, and before long, It became the foremost superconducting material in high-field applications. It is used today to make wires for superconducting magnets in NMR instruments and accelerators, such as the International Thermonuclear Experimental Reactor (ITER). Ted rose in the ranks at Bell Laboratories, and from 1957 to 1968 , he served as the head of the LowTemperature Physics Department there.

The next station in Ted's ascent was Stanford University, where he moved in 1968. He has been working at Stanford ever since - over a half of a century. He was the Theodore and Sydney Rosenberg endowed Chair in Applied Physics 1968-1990, the Chair of the Department of Applied Physics 1975-1978, the Director of the Center for Materials Research 1977-1988, and since 1990, is a Professor Emeritus of Applied Physics. In 1999, honoring his leadership in materials research, Stanford University founded the Theodore H. Geballe Laboratory for Advanced Materials (GLAM). Today, it is Stanford's foremost interdisciplinary research center, hosting professors and students from Physics, Applied Physics, Chemistry, Materials Science, Electrical Engineering, and other departments-including Ted himself. 
In his seven decades of scientific pursuits, Ted has studied a wide range of materials, achieved many important research results, and left his mark on many subjects. His monumental scientific bibliography, enclosed as an appendix in this special issue, encompasses some 500 research papers. These have been cited massively - well over 20,000 times. He also wrote, together with Robert White, a classic book, "Long Range Order in Solids."

Ted has served the community in many ways, as a scientist, educator, supervisor, advisor, and philanthrope. He participated in many committees and boards, including the Presidential National Advisory Committee on Superconductors, the Department of Energy Council on Materials Science, and the National Research Council. He has educated and supervised generations of scientists. Many of these have stayed in the field of superconductivity and materials physics, reaching prominence of their own. Frank DiSalvo, Frances Hellman, John Talvacchio, Raynien Kwo, Chang-Beom Eom, Byungdu Oh, Jonathan Sun, Yuri Suzuki, Steve Arnason, Andy Kent, Daniel Worledge, Rich Howard, Bob Schwall, Jim Harper, Walter Lowe, Dave Fork, and Kirsten (Luther) Myers are Ted's former students. Many others have since spread out to different fields.

Ted was elected to the National Academy of Sciences in 1973. He is also a member of the American Academy of Arts and Sciences, a fellow of the American Physical Society and of the American Association for the Advancement of Science, etc.; the complete list of his honors and distinctions is too long to reproduce here [1-4].

In an occasion like this, it is customary and expected to include some personal notes. Usually, it is some homageor, in plain English, flattery — which however Ted dislikes. So, I will just recount a few lessons I have learned from him when I was in his group, long ago, because these have served me well ever since.

The first message was that in our field, materials physics, the stars tend to favor the one who owns the best samples. This is particularly true when dealing with complex materials, like cuprate superconductors, with many atoms in the unit cell and many phases in the ternary or quaternary phase diagrams. In the HTS literature, erroneous conclusions abound, and most frequently, they are attributable to multi-phase and inhomogeneous samples. To know what you are looking at, you ought to synthesize and characterize the material yourself - and it better be the best there is. I have learned this lesson the hard way and ever since have invested heavily in materials synthesis and characterization — not because this is my primary and natural interest, but because this seems to be a necessary step on the path to unraveling the intrinsic physics of cuprates and the like.

Second, once when we discussed some HTS papers with questionable interpretation, Ted said something like this, "Theories may come and go, but accurate experimental data live forever." The message was registered - as Einstein said, when you hear the truth, you know it. So, ever since, this has been the leitmotif of my research and paper writing. Not to be misunderstood, this is not meant to be disparaging of theorywithout it, there would be no science (in fact, my $\mathrm{PhD}$, plus some $50+$ papers, was all purely theoretical). All I am saying is that in my experimental papers, I try to make the measurements at the state-of-the art (or better) level of accuracy, report the data as measured, and only draw the conclusions that directly follow from the raw data. By Ted's dictum, such conclusions should be safe and long lasting. In contrast, if the reportedly experimental findings in fact hinge on a lot of data fitting, DFT calculations, modelling, etc., there is always a danger of ending up wrong. Every theory starts from some assumptions, and if one or more of these are not realized in nature, the inferences could be wrong. This razor cuts both ways. If the credibility of a theory hinges on the agreement with (some) experiments, it is not a good sign; good theories vouch for themselves.

Last but not the least, Ted was always saying that science is a marathon - and proved that in the field. It did happen to me to feel tired of failures in the lab, or of arguing with an unfair referee, or an uncritical author (with me now playing the referee). But then, I would remember Ted and be put to shamehe does not get tired or bored. The experimental evidence is in the two photographs enclosed below; each is worth a thousand words (Figs. 1 and 2).

Clearly, science is Ted's lifelong passion. So, what follows is an offering that he may relish: a collection of original research articles in the fields of common interest to him and to this journal's readers. The list of authors includes such

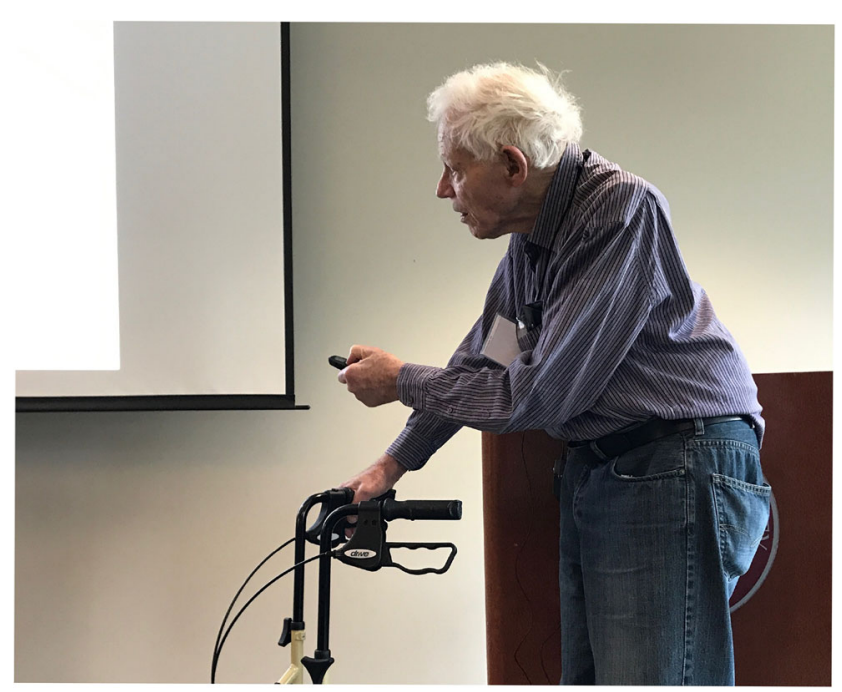

Fig. 1 Ted Geballe (97) delivering an invited talk at the conference "Towards Room Temperature Superconductivity" in Oxnard, California, organized by Armen Gulian. Ted's talk clocked at 45', plus questions and answers. The car ride Woodside-Oxnard is about $6 \mathrm{~h}$ one way - even with Jochen Manhart driving. But, as Ted would say, he was young(er) then (photographed by I.B. on May 09, 2017) 


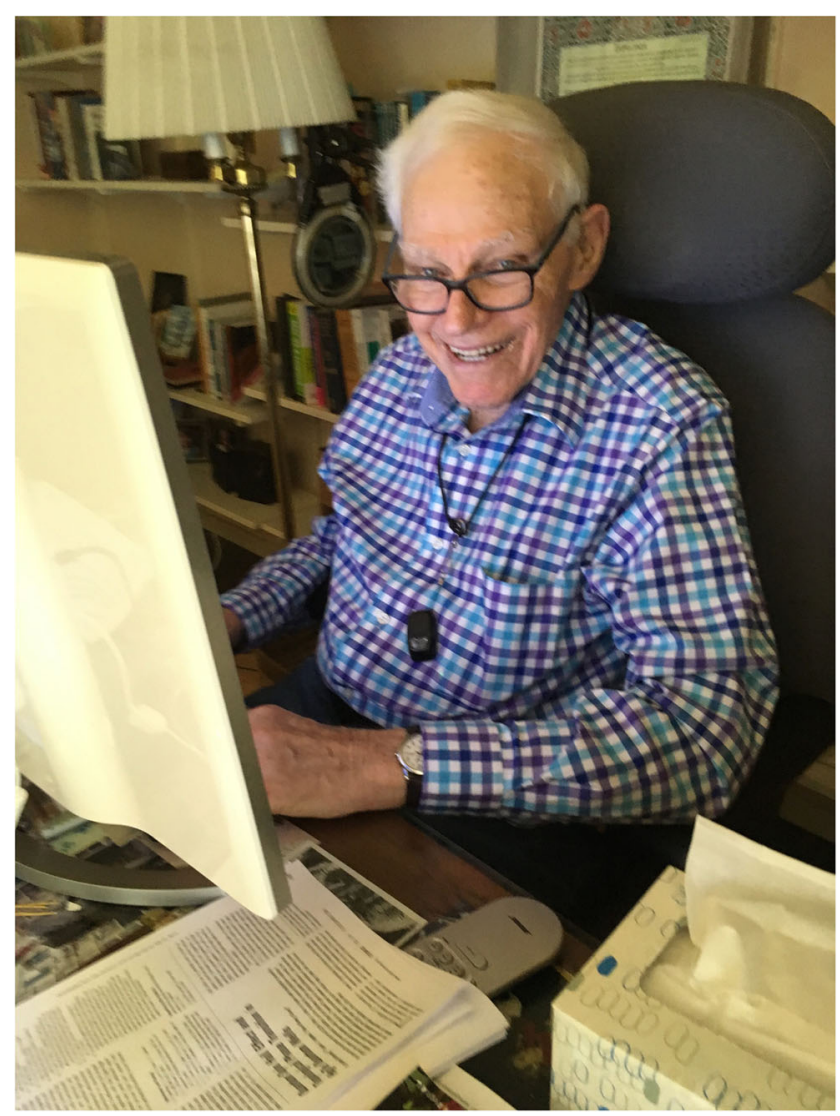

Fig. 2 Ted Geballe (98) working in his study. On my way between two conferences, I stayed at Ted's home in Woodside, California. Coming from Long Island, New York, and still on the East Coast time, I woke up $3 \mathrm{~h}$ too early. I walked around quietly not to wake up my hosts but found Ted in his office-already working. The paper he is studying is a hard-core theoretical stuff- "Quantum Spin Hall Effect and Topological Phase Transition in HgTe Quantum Wells," Science 314, 1757-1761 (2006), by Andrei Bernevig, Taylor Hughes, and Shou-Cheng Zhang (photographed by I.B. on July 28, 2018) eminent scientists as Bob Birgeneau, Doug Scalapino, Jim Phillips, Allen Goldman, Steve Kivelson, Jochen Mannhart, George Crabtree, and Andy Mackenzie. The Editors are grateful to all of them; we are aware that it was not easy to squeeze into their busy schedules the time to write the articles included in this collection. Their reward is that Ted will undoubtedly read all of these with a great interest and keen mind; we will not be surprised if he follows up and engages the authors with incisive comments, profound questions, and creative suggestions. More broadly, to the readership of the Journal of Superconductivity and Novel Magnetism, we hope that this special issue will be an inspiring and exciting reading.

Ted has lived to be a hundred. What we wish him next is to hold a room temperature superconductor in his hand-and smile.

\section{References}

1. Božović, I.: Theodore H. Geballe at 90. J Supercond Nov Magn. 23, 1419-1420 (2010)

2. Geballe, T.H.: Why I haven't retired. Annu Rev Cond Matter Phys. 4, 1-21 (2013)

3. Stewart, G.R.: Ted Geballe: a lifetime of contributions to superconductivity. Physica C. 514, 345-346 (2015)

4. Geballe, T.:"Family, friends, and physics" (Editions One Books. Berkeley, CA (2019).

Publisher's note Springer Nature remains neutral with regard to jurisdictional claims in published maps and institutional affiliations. 